\title{
Molecular gas toward G18.8+1.8
}

\author{
J. Vasquez ${ }^{1,2, \star \star}$, M. Rubio ${ }^{3}$, C. E. Cappa ${ }^{1,2, \star \star}$, and N. U. Duronea ${ }^{2}$ \\ ${ }^{1}$ Facultad de Ciencias Astronómicas y Geofísicas, Universidad Nacional de La Plata, Paseo del Bosque s/n, 1900 La Plata, Argentina \\ e-mail: pete@iar.unlp.edu.ar \\ 2 Instituto Argentino de Radioastronomía, CC 5, 1894 Villa Elisa, Argentina \\ 3 Departamento de Astronomía, Universidad de Chile, Casilla 36-D, Santiago, Chile
}

Received 1 March 2012 / Accepted 17 April 2012

\begin{abstract}
Aims. This work aims at investigating the characteristics of the molecular gas associated with the nebula G18.8+1.8, which is linked to the Wolf-Rayet star HD 168206 (WR 113), and its relation to other components of its local interstellar medium.

Methods. We carried out molecular observations of the ${ }^{12} \mathrm{CO}(J=1-0)$ and $(J=2-1)$ lines with an angular resolution of $44^{\prime \prime}$ and $22^{\prime \prime}$ using the SEST telescope. Complementary NANTEN data of the ${ }^{12} \mathrm{CO}(1-0)$ line were also used. The dust emission was analyzed using Spitzer-IRAC images at $8.0 \mu \mathrm{m}$, and WISE data at $3.4 \mu \mathrm{m}, 4.6 \mu \mathrm{m}$, and $12.0 \mu \mathrm{m}$.

Results. The SEST data allowed us to identify a molecular component (Cloud 3) that has velocities in the interval from $\sim+30$ to $+36 \mathrm{~km} \mathrm{~s}^{-1}$ and is most probably linked to the nebula. Morphological and kinematical properties suggest that Cloud 3 consists of a wind-blown molecular half-shell, which expands around WR 113. The ratio $R_{2-1 / 1-0}$ and excitation temperatures indicate that the molecular gas is being irradiated by strong UV radiation. The location of the inner optical ring in the outer edge of Cloud 3 suggests that the stars SerOB2-1, $-2,-3,-63$, and -64 are responsables for the ionization of Cloud 3 and the inner ring nebula. A comparison between the spatial distribution of the molecular gas and the PAH emission at $8 \mu$ m indicates the existence of a photodissociation region between the ionized and the molecular gas. A search for candidate young stellar objects (YSOs) in the region around G18.8+1.8 based on available 2MASS, MSX, IRAS, and Spitzer-IRAC catalogs resulted in the detection of about sixty sources, some of them projected onto Cloud 3. Two small spots of clustered candidate YSOs are projected near the outer border of Cloud 3, although a triggered stellar formation scenario is doubtful.
\end{abstract}

Key words. stars: Wolf-Rayet - ISM: bubbles - ISM: individual objects: G18.8+1.8 - stars: individual: WR 113

\section{Introduction}

Most massive star formation in our Galaxy occurs in giant molecular clouds (GMC) where, in general, the star formation is developed in groups like stellar clusters or OB associations. These groups can contain hundreds of OB stars, including massive young stellar objets (MYSOs) and evolved massive stars. Since OB associations directly influence the evolution of their environs, it is important to study how this interaction occurs in different interstellar environments. Massive stars interact with the interstellar medium (ISM) through their intense ultraviolet (UV) radiation field $(h v>13.6 \mathrm{eV})$ and via strong stellar winds. Ultraviolet photons ionize the surrounding gas that creates $\mathrm{H}$ II regions and dissociates the molecular gas from photodissociation regions (PDRs) in which it originated, while the second mechanism alters the structure of H II regions by sweeping up the circumstellar material and creating interstellar bubbles (IB) (Cappa et al. 2005).

SerOB2 is a rich OB association located at RA, $\operatorname{Dec}(\mathrm{J} 2000) \simeq\left(18^{\mathrm{h}} 18^{\mathrm{m}} 00^{\mathrm{s}},-11^{\circ} 36^{\prime} 36^{\prime \prime}\right)$, at a distance of $1.9 \pm 0.3 \mathrm{kpc}$ (Forbes 2000), about $70 \mathrm{pc}$ above the Galactic plane. Forbes (2000) performed a very interesting spectrophotometric study of this association and analyzed its connection to a narrow column of hot gas emerging from the H II region Sh2-54 perpendicularly to the Galactic plane, known as the thermal

\footnotetext{
* Appendix A is available in electronic form at

http://www . aanda.org

$\star \star$ Member of Carrera del Investigador, CONICET, Argentina.
}

"chimney" (Mueller et al. 1987). From the original set of stars $(\sim 500)$ taken for the analysis, only 107 were considered to be probable members of Ser OB2 (see Table 5 from Forbes 2000). Ser OB2 contains several massive stars, including the eclipsing binary system CV Ser (Massey \& Niemela 1981), the Oftype binary HD 166734 (Conti et al. 1980), the O-type multiple star HD 167971 (MY Ser; Leitherer et al. 1987), and the O5.5Vf star HD 168112.

Our aim in this paper is to investigate the existence of molecular gas in the environs of CV Ser and other posible members of Ser OB2 based on high angular resolution SEST observations performed in the ${ }^{12} \mathrm{CO}(1-0)$ and ${ }^{12} \mathrm{CO}(2-1)$ lines, complemented with medium angular resolution $\mathrm{CO}(1-0)$ data from the NANTEN telescope and infrared images in the mid-infrared (MIR).

\subsection{Background of CV Ser and its close environs}

The brightest component of CVSer is the Wolf-Rayet star WR 113 (三HD 168206, WC8d+O8-9IV, van der Hucht 2001). Table 1 summarizes the main parameters of WR 113: coordinates, spectral classification, visual absorption $A_{v}$, spectrophotometric distance, mass loss rate $\dot{M}$, and terminal velocity $v_{\infty}$.

Gonzalez \& Rosado (1984) discovered a double optical structure of $4^{\prime}$ and $9^{\prime}$ in radius around the WR star and suggested that the outer ring, which is poorly defined, was formed by the WR star or its massive progenitor and is presently being photoionized by the O5.5Vf star HD 168112 


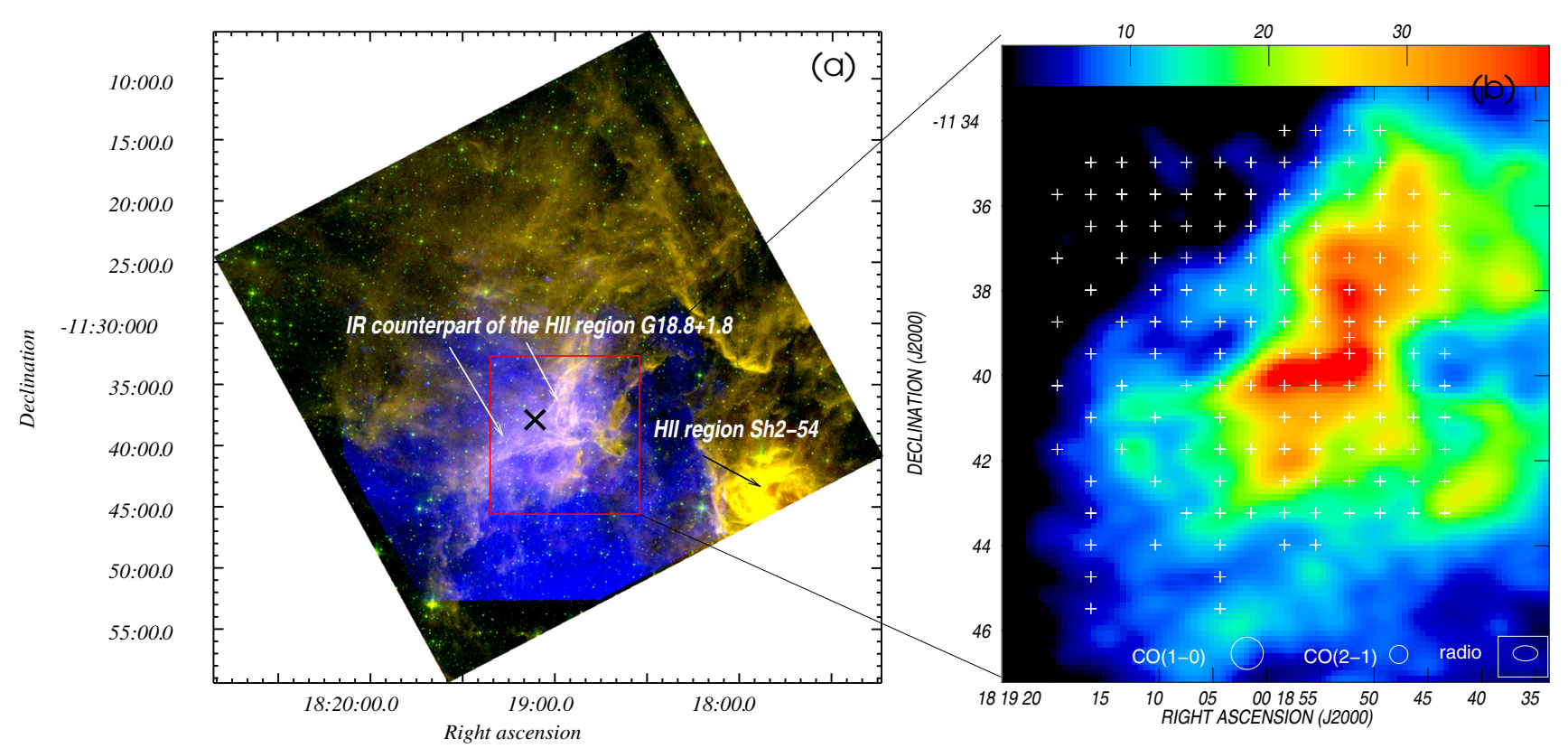

Fig. 1. Left panel: superposition of the IRAC images at $8.0 \mu \mathrm{m}$ (red) and $5.6 \mu \mathrm{m}$ (green) with the $\mathrm{H} \alpha$ emission (blue), of a section of the $\mathrm{H}$ II region Sh2-54 including the environs of WR 113. The cross marks the position of the WR star. Right panel: VLA image at $1465 \mathrm{MHz}$ showing the ionized ring. The crosses mark the positions of the 143 points observed in ${ }^{12} \mathrm{CO}$ lines. The color scale goes from 1 to $40 \mathrm{mJy}^{\text {beam }}{ }^{-1}$. The synthesized beam of the radio continuum observations and the beams of the molecular data are indicated in the bottom part of the image.

(at RA, Dec $(\mathrm{J} 2000)=\left(18^{\mathrm{h}} 18^{\mathrm{m}} 40.8^{\mathrm{s}},-12^{\circ} 06^{\prime} 23.4^{\prime \prime}\right)$, also belonging to Ser OB2 and located to the southwest of the ring nebula. Esteban \& Rosado (1995) observed the inner ring in $[\mathrm{NII}] \lambda \lambda 6548,6584,[\mathrm{SII}] \lambda \lambda 6717,6731, \mathrm{H} \alpha \lambda 6563$, and $\mathrm{H} \beta \lambda 4861$. They determined that the velocity of the ionized gas is in the range $[\sim+27, \sim+39] \mathrm{km} \mathrm{s}^{-1}$, which corresponds to a kinematical distance of $2.0 \pm 0.2 \mathrm{kpc}$, according to circular galactic rotation models (e.g. Brand \& Blitz 1993). A similar velocity of $+27 \mathrm{~km} \mathrm{~s}^{-1}$ was found from radio recombination lines by Lockman (1989). Esteban \& Rosado (1995) concluded that photoionization is the main source of excitation of the inner ring, for which the WR star is mainly responsible. Following the classification by Chu (1991) for WR ring nebulae, they classified the inner nebula as $R_{\mathrm{S}}$ type, because it does not present evidence of expansion and its morphology is shell-structured.

The left panel of Fig. 1 shows a superposition of the IRAC images at $8.0 \mu \mathrm{m}$ (in red) and $5.6 \mu \mathrm{m}$ (green), with the $\mathrm{H} \alpha$ emission (blue) of the ring nebula linked to CV Ser. The strong emission at RA, $\operatorname{Dec}(\mathrm{J} 2000)=\left(18^{\mathrm{h}} 18^{\mathrm{m}} 00^{\mathrm{s}},-11^{\circ} 44^{\prime}\right)$ corresponds to the H II region Sh2-54. The bright filament to the west and south of the binary system, which is indicated by the cross symbol, corresponds to the inner structure of $4^{\prime}$ in radius. This structure displays strong emission both in the $\mathrm{H} \alpha$ line and in the mid-infrared.

The right panel of Fig. 1 displays the radio continuum emission distribution at $1465 \mathrm{MHz}$, as observed by Cappa et al. (2002) using the Very Large Array (VLA) in DnC configuration with a synthesized beam of $30^{\prime \prime}$. The radio continuum source was first identified as G18.8 +1.8 by Goss \& Day (1970) at $2.7 \mathrm{GHz}$. The high angular resolution VLA data allowed them to detect radio counterparts of both the inner and outer rings. Adopting a distance of $2.0 \pm 0.6 \mathrm{kpc}$, Cappa et al. (2002) obtained ionized masses and electron densities of $20 \pm 10 M_{\odot}$ and $180-500 \mathrm{~cm}^{-3}$, respectively, for the inner shell, and $90 M_{\odot}$ and $40 \mathrm{~cm}^{-3}$, respectively, for the outer shell. From the IR-radio
Table 1. Main parameters of WR 113.

\begin{tabular}{lc}
\hline \hline RA, Dec $(\mathrm{J} 2000)$ & $\left(18^{\mathrm{h}} 19^{\mathrm{m}} 7.36^{\mathrm{s}},-11^{\circ} 37^{\prime} 59^{\prime} \cdot 20\right)$ \\
Spectral classification & WC8d $+\mathrm{O} 8-\mathrm{O}$ IV \\
$A_{v}(\mathrm{mag})$ & $3.23 \pm 0.1^{a}$ \\
$d(\mathrm{kpc})$ & $1.8^{a}, 2.0^{b}, 2.5^{e, f}$ \\
$\dot{M}\left(10^{-5} M_{\odot} \mathrm{yr}^{-1}\right)$ & $<5.6^{g}, 2.0 \pm 0.3^{h}, 2.4^{f}$ \\
$v_{\infty}\left(\mathrm{km} \mathrm{s}^{-1}\right)$ & $1400^{i}, 1890^{j}$ \\
\hline
\end{tabular}

References. (a) van der Hucht (2001), (b) Conti \& Vacca (1990), ${ }^{(e)}$ Esteban \& Rosado (1995), ${ }^{(f)}$ Nugis \& Lamers (2000), ${ }^{(g)}$ Leitherer et al. (1997), (h) Lamontagne et al. (1996), ${ }^{(i)}$ Koesterke \& Hamann (1995), ${ }^{(j)}$ Nugis et al. (1998).

continuum relation, they confirmed the thermal nature of the nebula. Adopting a radius $R_{\mathrm{S}}=2.3 \mathrm{pc}$ for the inner ring and an expansion velocity $v_{\exp }=5-10 \mathrm{~km} \mathrm{~s}^{-1}$, the derived dynamical age $t_{\mathrm{d}}=(1.3-2.5) \times 10^{5} \mathrm{yr}$ suggests that the inner shell originated in the action of the stellar wind of the current WR phase.

A search for OB stars within a region $12^{\prime}$ in size centered on the WR star indicated that other excitation sources are present in this region in addition to the binary system. Ser OB2-1, -2, and -3 are confirmed members of the $\mathrm{OB}$ association, while Ser OB2-63, and -64 are potencial members (see Table 5 from Forbes 2000). Their coordinates and $(B-V)$ and $(U-B)$ colors are listed in Table 2.

In our study, in agreement with Forbes (2000), we adopt a distance of $2.0 \pm 0.6 \mathrm{kpc}$. This value also agrees with the cataloged distances of the WR star (see Table 1).

\section{SEST observations and complementary data}

High angular resolution ${ }^{12} \mathrm{CO}(J=1 \rightarrow 0)$ and ${ }^{12} \mathrm{CO}(J=$ $2 \rightarrow 1$ ) data at 115 and $230 \mathrm{GHz}$, respectively, were obtained 
Table 2. Other excitation sources identified in the environs of CV Ser.

\begin{tabular}{lcccc}
\hline \hline & RA J(2000) & Dec J(2000) & $(B-V)$ & $(U-B)$ \\
& & & & \\
\hline Ser OB2-1 & 181836.9 & -114057.8 & 1.32 & 0.01 \\
Ser OB2-2 & 181839.9 & -114307.3 & 1.27 & 0.06 \\
Ser OB2-3 & 181842.4 & -114354.8 & 1.25 & 0.11 \\
Ser OB2-63 & 181853.4 & -114410.1 & 0.45 & 0.30 \\
Ser OB2-64 & 181905.0 & -114258.3 & 0.81 & 0.22 \\
\hline
\end{tabular}

Notes. From Forbes (2000).

with the $15 \mathrm{~m}$ Swedish-European Submillimetre Telescope (SEST), at La Silla, Chile during two observing runs in February 2002 and March 2003. The half-power beam-width of the telescope was $44^{\prime \prime}$ and $22^{\prime \prime}$ at 115 and $230 \mathrm{GHz}$, respectively. A high-resolution acousto-optical spectrometer was used. It consisted of 1000 chanels, with a total bandwidth of $100 \mathrm{MHz}$ and a resolution of $40 \mathrm{KHz}$, giving velocity resolutions of $0.105 \mathrm{~km} \mathrm{~s}^{-1}$ at $115 \mathrm{GHz}$ and $0.052 \mathrm{~km} \mathrm{~s}^{-1}$ at $230 \mathrm{GHz}$. The system temperatures were $\approx 400 \mathrm{~K}$ at $230 \mathrm{GHz}$ and $\approx 320 \mathrm{~K}$ at $115 \mathrm{GHz}$. Pointing was checked once during each observing run on the $\mathrm{SiO}(v=1$, $J=2 \rightarrow 1$ ) source VX Sgr. Pointing errors were $3^{\prime \prime}$. The uncertainty on the intensity calibration was $10 \%$. Details about the telescope and receivers can be found in Booth et al. (1989).

The ${ }^{12} \mathrm{CO}(2-1)$ and ${ }^{12} \mathrm{CO}(1-0)$ data were acquired simultaneously in the position-switching mode on a grid with a spacing of $45^{\prime \prime}$. The off-source position, at which no CO emission was detected, was placed at RA, Dec(J2000) = $\left(18^{\mathrm{h}} 19^{\mathrm{m}} 15^{\mathrm{s}},-11^{\circ} 44^{\prime}\right)$. A total of $143^{12} \mathrm{CO}$ spectra were taken toward the inner ring and its environs. The observed positions are indicated by crosses on the VLA image (Fig. 1, right panel).

The spectra were reduced using the CLASS software (GILDAS working group) ${ }^{1}$. The line intensities are expressed as main-beam brightness temperatures $T_{\mathrm{mb}}$, by dividing the antenna temperature $T_{\mathrm{A}}^{*}$ by the main-beam efficiency $\eta_{\mathrm{mb}}$, equal to 0.72 and 0.57 at 115 and $230 \mathrm{GHz}$, respectively (Johansson et al. 1998). The angular resolution of the ${ }^{12} \mathrm{CO}(2-1)$ and ${ }^{12} \mathrm{CO}(1-0)$ spectra, the velocity range, the original velocity resolution, the velocity resolution after smoothing, and the typical rms noise temperatures are listed in Table 3. ${ }^{12} \mathrm{CO}(2-1)$ and ${ }^{12} \mathrm{CO}(1-0)$ data cubes were constructed using the AIPS software.

Complementary ${ }^{12} \mathrm{CO}(1-0)$ data obtained with the 4-m NANTEN millimeter-wave telescope of Nagoya University were used to investigate the large-scale distribution of the molecular gas in a large area including SerOB2 and the WR star. The halfpower beamwidth was 2!6. The $4 \mathrm{~K}$ cooled SIS mixer receiver provided typical system temperatures of $\approx 220 \mathrm{~K}(\mathrm{SSB})$ at this frecuency. The acoustoptical spectrometer (AOS) provided a velocity coverage range of $100 \mathrm{~km} \mathrm{~s}^{-1}$ and a velocity resolution of $1.0 \mathrm{~km} \mathrm{~s}^{-1}$.

The distribution of the IR emission was analyzed using Spitzer images at 5.6 and $8.0 \mu \mathrm{m}$ from the Galactic Legacy Infrared Mid-Plane Survey Extraordinaire (Spitzer-GLIMPSE, Benjamin et al. 2003) retrieved from the Spitzer Science Center ${ }^{2}$. Images at $3.4,4.6$, and $12.0 \mu \mathrm{m}$ with angular resolutions of 6.' 1 , 6.'5, and 6.'5 from the Wide-field Infrared Survey Explorer (WISE; Wright et al. 2010) were retrieved from IPAC ${ }^{3}$.

\footnotetext{
${ }^{1}$ http://www.iram.fr/IRAMFR/PDB/class/class.html

2 http://scs.spitzer.caltech.edu

${ }^{3}$ http://www.ipac. caltech.edu
}

Table 3. Observational parameters of the ${ }^{12} \mathrm{CO}(1-0)$ and ${ }^{12} \mathrm{CO}(2-1)$ lines observed with SEST.

\begin{tabular}{lcc}
\hline \hline & ${ }^{12} \mathrm{CO}(2-1)$ & ${ }^{12} \mathrm{CO}(1-0)$ \\
\hline Angular resolution (") & 22 & 45 \\
Velocity range $\left(\mathrm{km} \mathrm{s}^{-1}\right)$ & $(-7,+47)$ & $(-40,+70)$ \\
$\begin{array}{l}\text { Original velocity } \\
\text { Resolution }\left(\mathrm{km} \mathrm{s}^{-1}\right)\end{array}$ & 0.054 & 0.110 \\
$\begin{array}{l}\text { Velocity resolution } \\
\text { After smoothing }\left(\mathrm{km} \mathrm{s}^{-1}\right)\end{array}$ & 0.221 & 0.332 \\
rms noise (K) & 0.17 & 0.25 \\
\hline
\end{tabular}

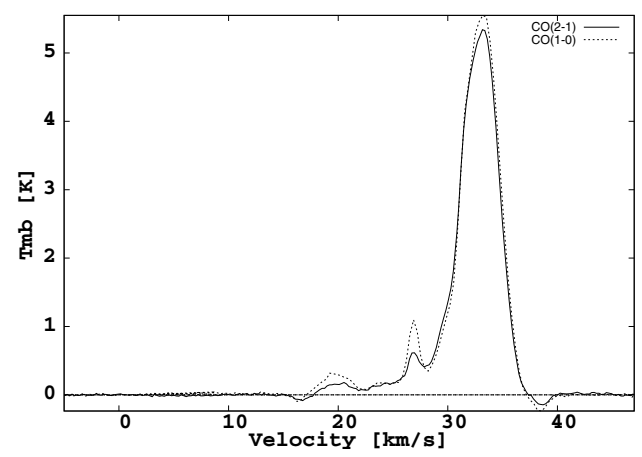

Fig. 2. ${ }^{12} \mathrm{CO}(2-1)$ and ${ }^{12} \mathrm{CO}(1-0)$ spectra averaged within the surveyed region. Line intensities are expressed as main-beam brightness temperature $T_{\mathrm{mb}}$. Velocities are referred to the LSR.

\section{The characteristics of the molecular gas}

\subsection{Spatial distribution}

Aimed at detecting the molecular gas that is possibly linked to the ring nebula, we have inspected the data cubes of the ${ }^{12} \mathrm{CO}(2-1)$ and ${ }^{12} \mathrm{CO}(1-0)$ lines obtained with SEST. The ${ }^{12} \mathrm{CO}(2-1)$ and ${ }^{12} \mathrm{CO}(1-0)$ spectra averaged within the surveyed region are displayed in Fig. 2. Carbon monoxide emission higher than $5 \mathrm{rms}$ is detected between +18 and $+37 \mathrm{~km} \mathrm{~s}^{-1}$. Three molecular components are noticed within the mentioned velocity interval, peaking at $\sim+20 \mathrm{~km} \mathrm{~s}^{-1}, \sim+28 \mathrm{~km} \mathrm{~s}^{-1}$, and $+34 \mathrm{~km} \mathrm{~s}^{-1}$. In Fig. 3 (left panels) we show the spatial distribution of the molecular gas in the velocity ranges from +18.6 to $+22.2 \mathrm{~km} \mathrm{~s}^{-1}$ (Cloud 1), from +25.8 to $+28.9 \mathrm{~km} \mathrm{~s}^{-1}$ (Cloud 2), and from +30 to $+36.8 \mathrm{~km} \mathrm{~s}^{-1}$ (Cloud 3 ) obtained from SEST data. CO intensities are expressed as main-beam brightness temperature averaged within the selected velocity intervals. The cross indicates the position of the WR star. To illustrate the large-scale distribution of these molecular clouds, the molecular emission in the same velocity ranges as obtained with the NANTEN data is shown in the right panels of Fig. 3, superimposed onto the DSSR image.

Cloud 1 is elongated in the north-south direction with its brightest emission at RA, Dec $(\mathrm{J} 2000) \sim\left(18^{\mathrm{h}} 18^{\mathrm{m}} 50^{\mathrm{s}},-11^{\circ} 39^{\prime}\right)$. A fainter clump is also detected at RA, Dec(J2000) $\left(18^{\mathrm{h}} 18^{\mathrm{m}} 57^{\mathrm{s}},-11^{\circ} 37^{\prime}\right)$. The NANTEN image shows that this cloud is projected onto the western border of the optical nebula and has a very small angular extension. Using the analytical fit to the circular galactic rotation model of Brand \& Blitz (1993) along $l \approx 20^{\circ}$ we derived for Cloud 1 near and far kinematical distances of about 2 and $14 \mathrm{kpc}$, respectively. Bearing in mind the lack of morphological agreement with the optical nebula and its small angular size, we believe that Cloud 1 may be a background object, unrelated to the star and the ionized regions. A distance as far as $\sim 14 \mathrm{kpc}$ cannot be ruled out. 

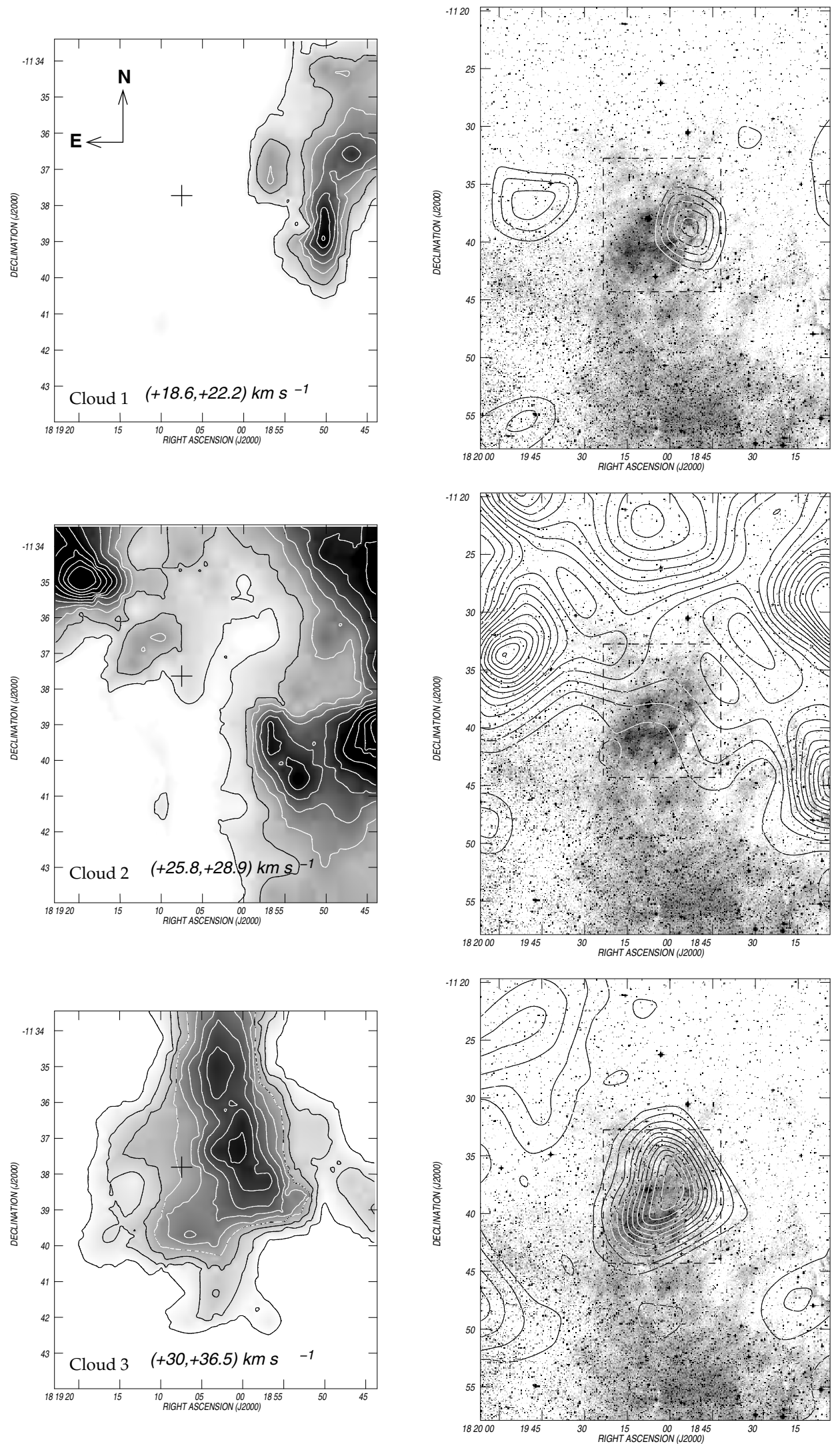

Fig. 3. Left panels: ${ }^{12} \mathrm{CO}(1-0)$ emission distribution maps corresponding to Clouds 1 to 3 obtained with the SEST data. For Cloud 1 , the grayscale goes from 0.2 to $2 \mathrm{~K}$, and the contour levels are from $0.3 \mathrm{~K}(\sim 4.5 \mathrm{rms})$ in steps of $0.3 \mathrm{~K}$. For Cloud 2, the grayscale goes from 0.2 to $0.25 \mathrm{~K}$, and the contour levels are from $0.3 \mathrm{~K}(\sim 4 \mathrm{rms})$ in steps of $0.3 \mathrm{~K}$. For Cloud 3, the grayscale goes from 1 to $8 \mathrm{~K}$, and the contour levels goes from $1 \mathrm{~K}$ $(\sim 20 \mathrm{rms})$ in steps of $1 \mathrm{~K}$. The cross indicates the position of the WR star. Right panels: ${ }^{12} \mathrm{CO}(1-0)$ emission distribution maps corresponding to Clouds 1 to 3 obtained with the NANTEN data (contours), superimposed onto the DSSR image (grayscale). The dashed rectangles at the center of the field indicate the area of the SEST images at the left. 
Cloud 2 displays a very clumpy morphology. Although it encircles most of the optical nebula, it shows no clear morphological agreement with the ionized gas. Its maxima are present at RA, Dec $(\mathrm{J} 2000) \sim\left(18^{\mathrm{h}} 18^{\mathrm{m}} 55^{\mathrm{s}},-11^{\circ} 40^{\prime}\right), \mathrm{RA}, \operatorname{Dec}(\mathrm{J} 2000) \sim$ $\left(18^{\mathrm{h}} 19^{\mathrm{m}} 13^{\mathrm{s}},-11^{\circ} 37^{\prime} 30^{\prime \prime}\right)$, and RA, Dec $(\mathrm{J} 2000) \sim\left(18^{\mathrm{h}} 19^{\mathrm{m}} 20^{\mathrm{s}}\right.$, $\left.-11^{\circ} 35^{\prime}\right)$. Cloud 2 matches regions without optical emission toward the northern, western, and southwestern sectors of the nebula. An inspection of the middle right panel of Fig. 3 clearly indicates that Cloud 2 extends well beyond the area surveyed with SEST and NANTEN, which suggests that this cloud is part of a giant molecular cloud (GMC).

The molecular emission of Cloud 3 appears to be mostly located in the region of the optical nebula. The bulk of the molecular gas is concentrated in an elongated feature extending from between RA, $\operatorname{Dec}(\mathrm{J} 2000) \sim\left(18^{\mathrm{h}} 19^{\mathrm{m}} 05^{\mathrm{s}},-11^{\circ} 34^{\prime}\right)$ to RA, Dec $(\mathrm{J} 2000) \sim\left(18^{\mathrm{h}} 19^{\mathrm{m}},-11^{\circ} 39^{\prime}\right)$. At lower declinations, this feature is shifted toward the east, encircling the position of the WR star. The size and morphology of Cloud 3 excellently agrees with the optical nebula. The NANTEN image corresponding to Cloud 3 shows that the bulk of the molecular gas of this cloud coincides with that of the optical nebula and does not extend beyond the region surveyed by the SEST data.

The large extent of Cloud 2 and the morphological coincidence with a region without optical emission toward the north and west of the nebula speaks in favor of an interpretation in which Cloud 2 is a foreground object with respect to WR 113, or is placed at almost the same distance. On the other hand, the good morphological correspondence of Cloud 3 with the inner optical nebula around WR 113 suggests that this structure is physically related to the ionized ring nebula. We point out that along this galactic longitude, considering mean radial velocities of about $\sim+27.5 \mathrm{~km} \mathrm{~s}^{-1}$ and $\sim+35.5 \mathrm{~km} \mathrm{~s}^{-1}$, the galactic velocity field of Brand \& Blitz (1993, see their Fig. 2b) along $l \approx 20^{\circ}$ predicts near kinematical distances of $1.8 \pm 0.5 \mathrm{kpc}$ and $2.3 \pm 0.5 \mathrm{kpc}$ for Cloud 2 and Cloud 3, respectively. Uncertainties on these distances were obtained adopting random motions of $\pm 6 \mathrm{~km} \mathrm{~s}^{-1}$ (Burton \& Gordon 1978). Keepeing this in mind, we cannot rule out an interpretation in which Cloud 3 is behind Cloud 2. Nevertheless, from the present data we cannot conclude about a physical relation between Cloud 2 and the optical nebula. Based on the above, from here onward we will concentrate on the analysis of Cloud 3 , which is the only cloud clearly associated with the optical ring nebula around WR 113.

To better characterize the kinematical properties of Cloud 3 , we show in Fig. 4 a collection of images depicting the $\mathrm{CO}(1-0)$ spatial distribution in the velocity range from +28.9 to $+36.9 \mathrm{~km} \mathrm{~s}^{-1}$. Every image displays the $\mathrm{CO}$ emission averaged over a velocity interval of $\sim 0.9 \mathrm{~km} \mathrm{~s}^{-1}$ (three individual smoothed channel maps). The $\mathrm{CO}$ emission distribution shown in Fig. 4 (in contours) is projected onto the DSSR image of the optical nebula (grayscale). The velocity interval of the individual images is indicated in the lower right corner. In the velocity interval from +29.8 to $+32.5 \mathrm{~km} \mathrm{~s}^{-1}$ the bulk of the molecular emission is concentrated toward the center of the optical nebula and is projected onto the region of relatively faint emission between the stellar position and the bright optical filaments. This coincidence suggests that the faint optical emission observed toward the center of the nebula arises from ionization of photodissociated molecular gas. The VLA image also shows low radio continuum emission in this area, indicating the presence of ionized gas. From $+32.5 \mathrm{~km} \mathrm{~s}^{-1}$ the molecular gas develops an arc-like morphology, which is better noticed at velocities between $\sim+34.3$ and +35.2 . This morphology remains at $36.0 \mathrm{~km} \mathrm{~s}^{-1}$ where it is still noticed as a hollow patchy annular feature.

\subsection{Ratio $R_{2-1 / 1-0}$ and excitation temperature}

A powerful tool that helps us in determining the physical conditions of molecular clouds is the $R_{2-1 / 1-0}$ ratio. Sakamoto et al. (1997) classified the molecular gas into three categories: "very high-ratio gas" (VHRG, corresponding to $1.0<R_{2-1 / 1-0}<1.2$ ), "high-ratio gas" (HRG, for $0.7<R_{2-1 / 1-0}<1$ ), and "low-ratio gas" (LRG, for $R_{2-1 / 1-0}<0.7$ ). Values compatible with VHRG denote surfaces of dense clumps irradiated by a strong radiation field and PDRs (Gierens et al. 1992). In this case, excitation temperatures $\left(T_{\text {exc }}\right)$ greater than $50 \mathrm{~K}$ and volume densities greater than $3 \times 10^{3} \mathrm{~cm}^{-3}$ are expected. Molecular gas with $R_{2-1 / 1-0} \simeq 1.3$ is observed toward $\mathrm{H}$ II regions (Castets et al. 1990; Sakamoto et al. 1994), which heat its surrounding molecular dense gas. Ratios in the range 0.7 to 1.0 are usually observed in central regions of giant molecular clouds where $\mathrm{CO}$ emission originates in collision processes (Castets et al. 1990; Sakamoto et al. 1994). This material is characterized by $T_{\text {exc }} \gtrsim 20 \mathrm{~K}$ and $n_{\mathrm{H}_{2}} \gtrsim 1 \times 10^{3} \mathrm{~cm}^{-3}$. Finally, $R_{2-1 / 1-0}<0.7$ is observed in the outer envelopes of giants molecular clouds. These values are consistent with subthermally excited gas, with $T_{\text {exc }} \leq 10 \mathrm{~K}$ and densities $n_{\mathrm{H}_{2}}<1 \times 10^{3} \mathrm{~cm}^{-3}$. To probe the surface conditions of Cloud 3, we analyzed the $T_{\text {exc }}$-distribution obtained from the $\mathrm{CO}(1-0)$ line, which is assumed to be optically thick.

Figure 5 shows the spatial distribution of the ratio $R_{2-1 / 1-0}$ obtained for Cloud 3 in grayscale, where

$R_{2-1 / 1-0}=\frac{\int_{v_{1}}^{v_{2}} T_{\mathrm{mb}(2-1)} \mathrm{d} v}{\int_{v_{1}}^{v_{2}} T_{\mathrm{mb}(1-0)} \mathrm{d} v}$

with $v_{1}=29 \mathrm{~km} \mathrm{~s}^{-1}$ and $v_{2}=36.5 \mathrm{~km} \mathrm{~s}^{-1}$. The spatial distribution of $\int_{v_{1}}^{v_{2}} T_{\mathrm{mb}(2-1)} \mathrm{d} v$ and $\int_{v_{1}}^{v_{2}} T_{m b}(1-0) \mathrm{d} v$ (order-zero moments) were obtained using AIPS package.

The $T_{\text {exc }}$ distribution of the ${ }^{12} \mathrm{CO}$ line can be obtained from $T_{\text {peak }}\left({ }^{12} \mathrm{CO}\right)=T^{*}\left[J_{v}\left(T_{\text {exc }}\right)-J_{v}\left(T_{\mathrm{bg}}\right)\right]($ Dickman 1978), where $T_{\text {peak }}$ is the peak temperature of the ${ }^{12} \mathrm{CO}(1-0)$ line, $T^{*}=h v / k$, with $v$ the rest frequency of the ${ }^{12} \mathrm{CO}(1-0)$ line, and $J_{v}(T)=\left(\exp \left(T^{*} / T\right)-1\right)^{-1}$. We adopt a background temperature $T_{\text {bg }} \sim 2.7 \mathrm{~K}$. Using this equation, assuming Gaussian profiles for the $\mathrm{CO}(1-0)$ line, and combining the order-zero moment map (i.e., integrated areas) with the order-two moment maps (i.e., velocity dispersion), we obtain the $T_{\text {exc }}$ distribution map, which is depicted in Fig. 5 with black contours.

An inspection of Fig. 5 shows values of $R_{2-1 / 1-0}$ between 0.5 and 1.2 along Cloud 3 . It is worth to mention that values of $R_{2-1 / 1-0}$ higher than 1.2 in the border of the $\mathrm{CO}$ cloud cannot be taken into account due to their large uncertainties. Two small regions at $18^{\mathrm{h}} 19^{\mathrm{m}} 07^{\mathrm{s}}<\mathrm{RA}<18^{\mathrm{h}} 19^{\mathrm{m}} 13^{\mathrm{s}}$ and $-11^{\circ} 37 .^{\prime} 5<\operatorname{Dec}<-11^{\circ} 39^{\prime}$, and at $18^{\mathrm{h}} 19^{\mathrm{m}} 05^{\mathrm{s}}<\mathrm{RA}<$ $18^{\mathrm{h}} 19^{\mathrm{m}} 12^{\mathrm{s}}$ and $-11^{\circ} 33 .^{\prime} 5<$ Dec $<-11^{\circ} 35 .^{\prime} 5$ exhibit values of $R_{2-1 / 1-0}$ between $\sim 0.4$ to $\sim 0.7$, probably arising in spots of quiescent dark molecular gas. The rest of the molecular cloud shows higher values (between $\sim 0.8$ and $\sim 1.1$ ), which suggests that the molecular gas of Cloud 3 is being irradiated by an external UV field and might be indicative of the presence of PDRs along Cloud 3. A similar conclusion can be reached from the $T_{\text {exc }}$-analysis. Excitation temperatures of about $\sim 50 \mathrm{~K}$ are observed toward the brightest part of Cloud 3, which are higher than expected inside molecular cores if only cosmic ray ionization is considered as the main heating source $(T \sim 8-10 \mathrm{~K}$, van der Tak \& van Dishoeck 2000). Excitation temperatures of about $\sim 30 \mathrm{~K}$ are observed toward molecular clouds located at the edges of evolved H II regions 


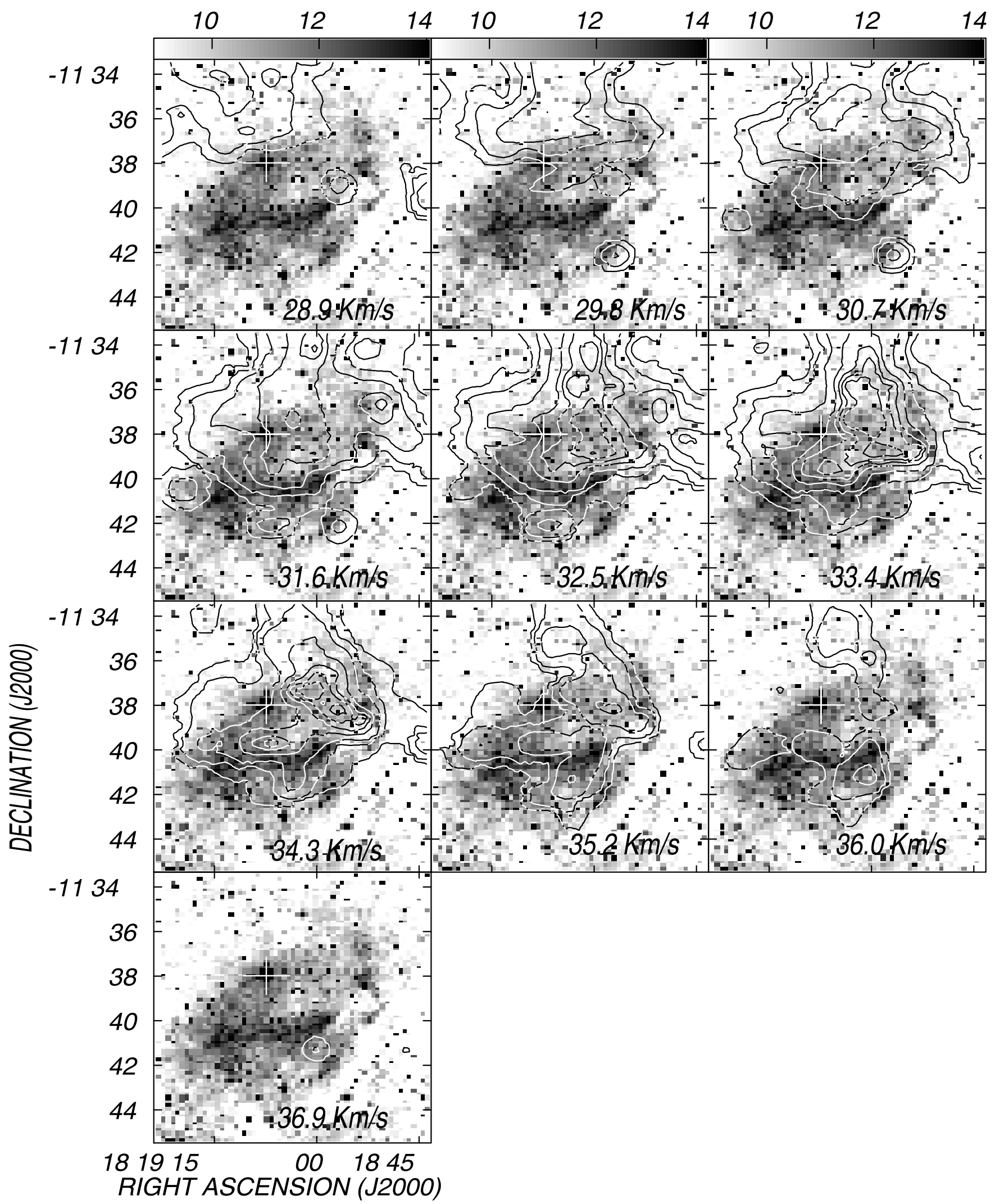

Fig. 4. Overlay of the mean CO emission (contours) in the velocity range from 28.9 to $36.9 \mathrm{~km} \mathrm{~s}^{-1}$ and the DSSR emission of the optical nebula (grayscale). The central velocity is indicated in the lower right corner of each image. Contour levels are 1, 2, 4, 8, 11, 13, and 15 K. The position of WR 113 is indicated with the white cross.

(bright rimmed clouds), which implies that additional heating processes, such as photoionization, are present close to these clouds (Urquhart et al. 2009). We conclude that Cloud 3 is being externally heated through the photoionization of its surface layers as a consequence of its proximity to neighboring ionizing stars. 


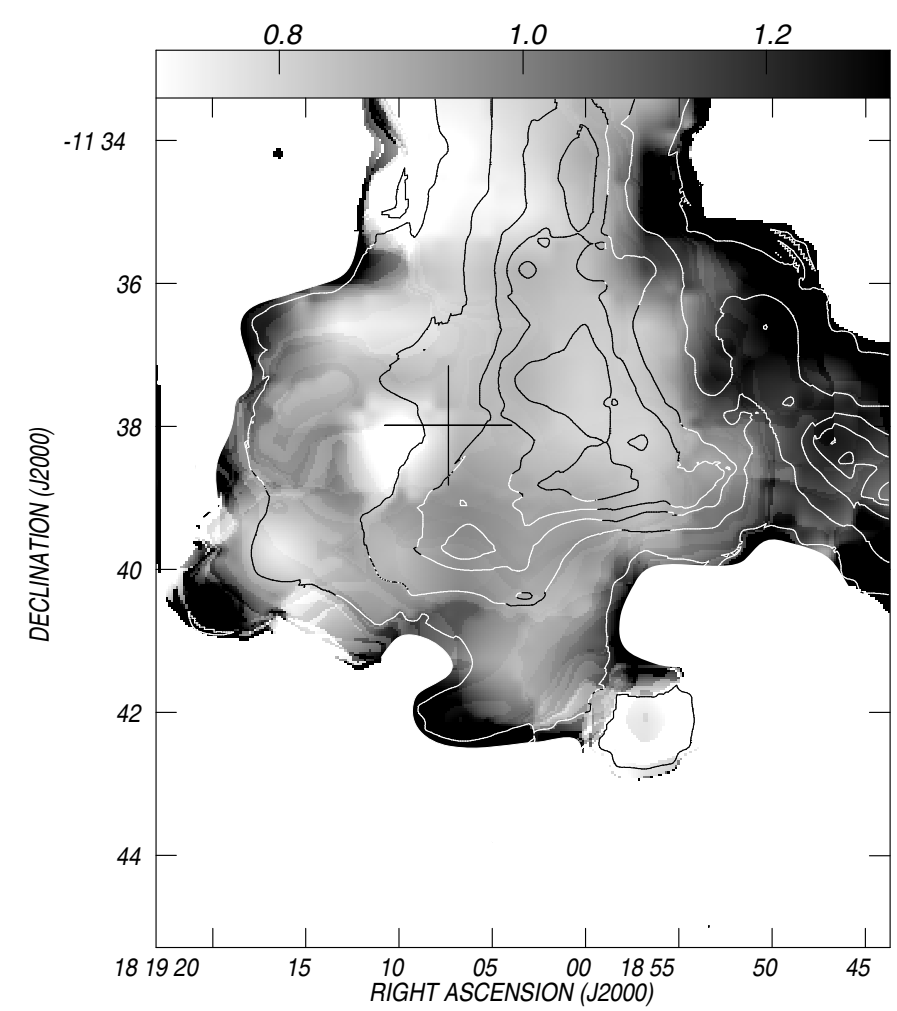

Fig. 5. Line ratios $R_{2-1 / 1-0}$ for Cloud 3 . The grayscale goes from $\sim 0.6$ (light gray) to $\sim 1.3$ (dark gray). Black contours correspond to the $T_{\text {exc }}$ of the ${ }^{12} \mathrm{CO}(1-0)$ line. $T_{\text {exc }}$ contours are $10,20,30,35,40$, and $50 \mathrm{~K}$.

Table 4. Main physical parameters of Cloud 3.

\begin{tabular}{lc}
\hline \hline Parameter & Value \\
\hline Angular size (') & $\sim 7$ \\
Linear size $(\mathrm{pc})$ & $4.0 \pm 1.2$ \\
Velocity range $\left(\mathrm{km} \mathrm{s}^{-1}\right)$ & $\sim+30,+36$ \\
Systemic velocity $\left(\mathrm{km} \mathrm{s}^{-1}\right)$ & $\sim 35.5 \pm 1.0$ \\
$\mathrm{H}_{2}$ column density $N_{\mathrm{H}_{2}}\left(10^{21} \mathrm{~cm}^{-2}\right)$ & $4.1 \pm 0.6$ \\
Molecular mass $M_{\mathrm{H}_{2}}\left(M_{\odot}\right)$ & $\sim 1600 \pm 500$ \\
$n_{\mathrm{H}_{2}}\left(\right.$ molecules $\left.\mathrm{cm}^{-3}\right)$ & $\sim 450$ \\
\hline
\end{tabular}

\subsection{Physical parameters of the molecular cloud}

Table 4 lists the main physical parameters of Cloud 3, obtained from the ${ }^{12} \mathrm{CO}(1-0)$ observations. The table includes angular sizes, velocity ranges, systemic velocities, mean $\mathrm{H}_{2}$ column densities, and molecular masses. Mean $\mathrm{H}_{2}$ column densities were derived using the empirical relation between the integrated emission $I_{\mathrm{CO}}\left(=\int T_{\mathrm{mb}} \mathrm{d} v\right)$ and $N_{\mathrm{H}_{2}}$. We adopted $N\left(\mathrm{H}_{2}\right)=$ $(1.9 \pm 0.3) \times 10^{20} I_{\mathrm{CO}}\left(\mathrm{cm}^{-2}\right)$ (Digel et al. 1996; Strong \& Mattox 1996). The total molecular mass was calculated using $M_{\mathrm{H}_{2}}=\left(m_{\text {sun }}\right)^{-1} \mu m_{\mathrm{H}} \sum \Omega N\left(\mathrm{H}_{2}\right) d^{2}\left(M_{\odot}\right)$, where $m_{\text {sun }}$ is the solar mass $\left(\sim 2 \times 10^{33} \mathrm{~g}\right), \mu$ is the mean molecular weight, assumed to be equal to 2.8 after allowance of a relative helium abundance of $25 \%$ by mass (Yamaguchi et al. 1999), $m_{\mathrm{H}}$ is the hydrogen atom mass $\left(\sim 1.67 \times 10^{-24} \mathrm{~g}\right), \Omega$ is the solid angle subtended by the $\mathrm{CO}$ feature in ster, and $d$ is the distance. The mean volume density $\left(n_{\mathrm{H}_{2}}\right)$ of Cloud 3 can be derived from the ratio of its molecular mass and its volume considering an approximately spherical geometry with a radious of about $2.3 \mathrm{pc}$.

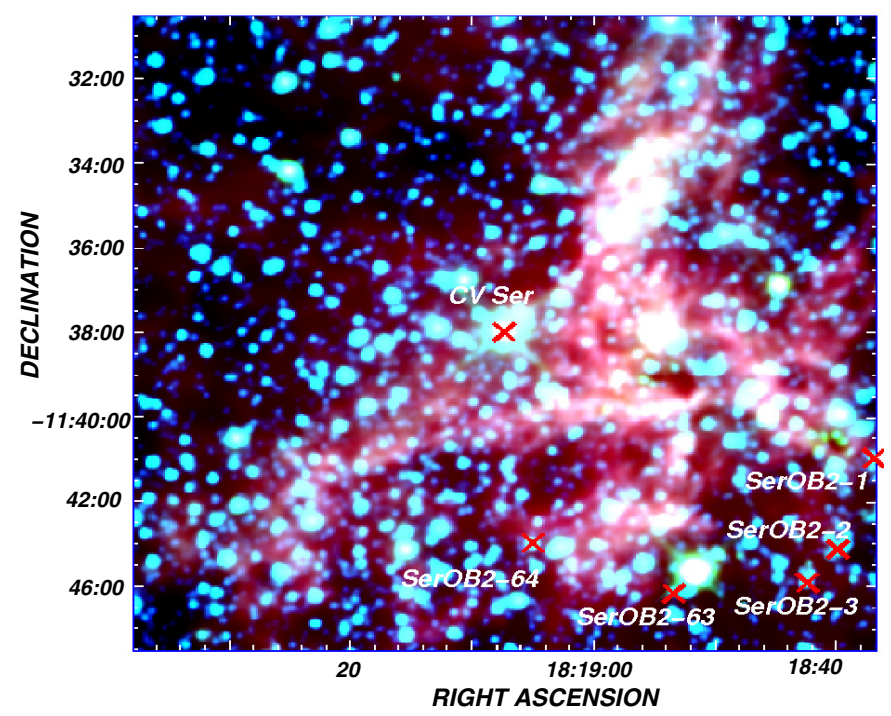

Fig. 6. Superposition of the MIR emissions at $3.4 \mu \mathrm{m}$ (blue), $4.6 \mu \mathrm{m}$ (green), and $12.0 \mu \mathrm{m}$ (red). The positions of WR 113 and the Ser OB2 members are indicated by red crosses.

\section{IR counterparts and photodissociated regions}

In Fig. 6 we show the mid infrarred (MIR) emission at $3.4 \mu \mathrm{m}$ (blue), $4.6 \mu \mathrm{m}$ (green), and $12.0 \mu \mathrm{m}$ (red) in the region of the inner ring nebula. The $3.4 \mu \mathrm{m}$ and $12 \mu \mathrm{m}$ filters include prominent PAH emission features and the $4.6 \mu \mathrm{m}$ filter measures the continuum emission from very small grains (Wright et al. 2010). The emision at $12.0 \mu \mathrm{m}$ resembles the observed emission at $8.0 \mu \mathrm{m}$ (see Fig. 1). The emission at $4.6 \mu \mathrm{m}$ is barely detected toward some stellar sources while the emission at 3.4 and $12 \mu$ m clearly delineates the inner optical ring.

Figure 7 shows the IR emission distribution from the SpitzerIRAC $8.0 \mu \mathrm{m}$ image (red), the optical DSS $2 \mathrm{R}$ image (blue), and the $\mathrm{CO}(2-1)$ contours over a field larger than the region surveyed with SEST. The emission in $8.0 \mu \mathrm{m}$, mainly originated in the strong 7.7 and $8.3 \mu \mathrm{m} \mathrm{PAH}$ features, shows a concentration of material along two elongated almost perpendicular regions placed at $\mathrm{RA} \sim 18^{\mathrm{h}} 18^{\mathrm{m}} 55^{\mathrm{s}}$, extending from Dec $\sim-11^{\circ} 40^{\prime}$ to $-11^{\circ} 30^{\prime}$, and at Dec $\sim-11^{\circ} 40^{\prime}$, from RA $\sim 18^{\mathrm{h}} 19^{\mathrm{m}} 30^{\mathrm{s}}$ to $18^{\mathrm{h}} 18^{\mathrm{m}} 50^{\mathrm{s}}$. As we described in Sect. 1.1, the optical inner bright filament appears to be bordering the MIR emission. Figure 7 also shows the diffuse optical emission north and south of the inner optical filament. Both emissions, optical and MIR, enclose the molecular cloud toward the south and west.

The presence of PAH emission, which is typical of PDRs, at the interphase between the ionized and molecular gas suggests that the molecular gas is being photodissociated by the UV photons emitted by the massive stars (Vasquez et al. 2010). For this reason and according to the morphology of the PAH features observed in Figs. 6 and 7, and the optical and molecular emissions (see Fig. 4), we can confirm the presence of a PDR located at Dec $\sim-11^{\circ} 41^{\prime}$, from RA $\sim 18^{\mathrm{h}} 18^{\mathrm{m}} 50^{\mathrm{s}}$ to $18^{\mathrm{h}} 19^{\mathrm{m}} 15^{\mathrm{s}}$, which could be generated mainly by the action of the photodissociating far-ultraviolet $(6 \mathrm{eV}<h v<13.6 \mathrm{eV})$ photons of Ser OB2-1, -2, -3, -63, and -64 .

\section{Star formation}

To investigate the presence of protostellar candidates in the region, we used data from the available MSX, 2MASS, IRAS, and 


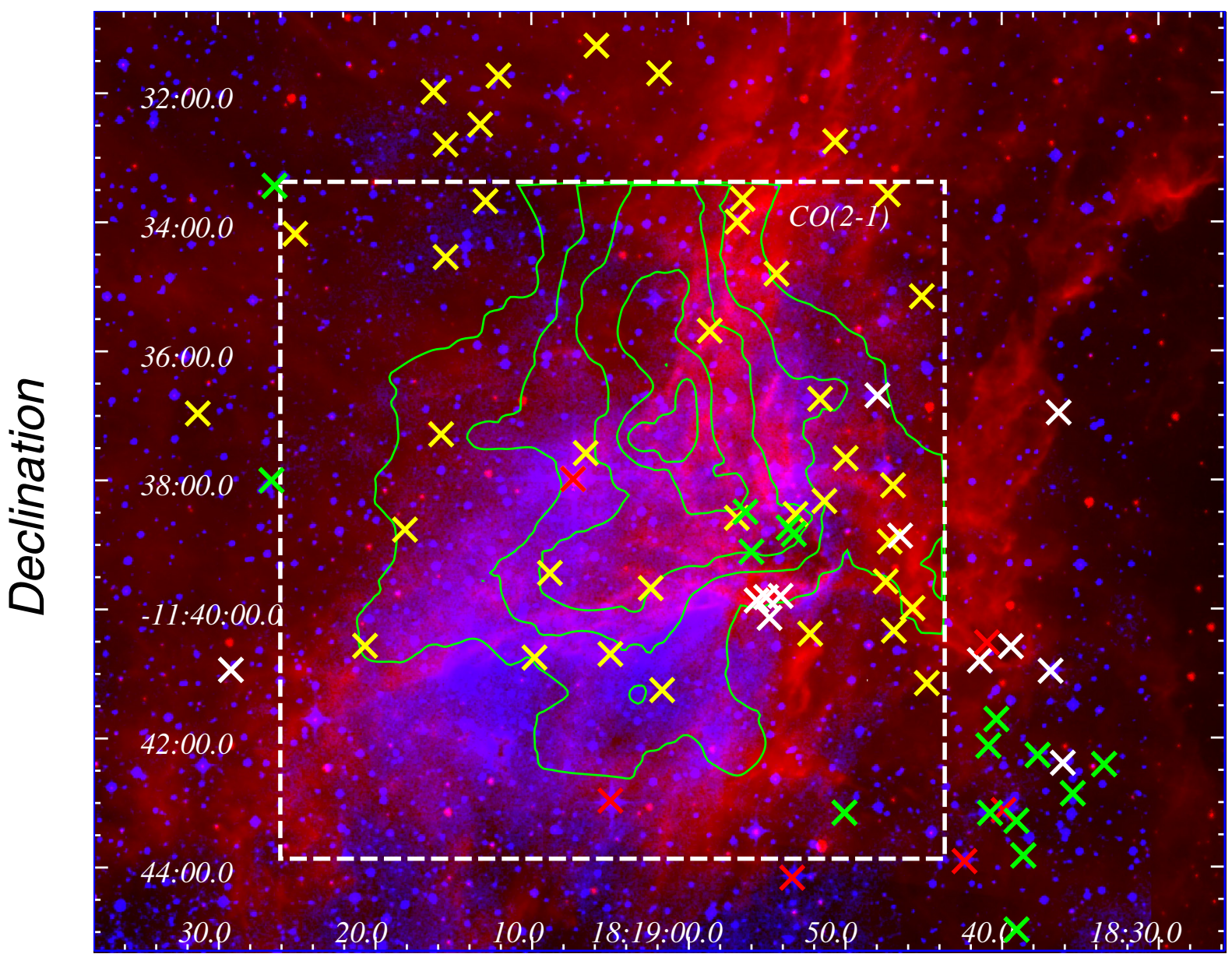

\section{Right Ascension (J2000)}

Fig. 7. Overlay of the $8 \mu \mathrm{m}$-image (in red), the optical (DSS2 R, in blue), and millimeter (CO(2-1), green contours) images of the region. The yellow crosses indicate the position of the 2MASS pointo sources with IR excess, while the white and green crosses indicate the IRAC class I and class II sources, respectively. Finally, the red crosses indicate the position of CV Ser and Ser OB-1, -2, -3, -63 and -64.

Spitzer-IRAC point source catalogs in a region of about $10^{\prime}$ in size centered on the position of the WR star.

From an original set of $\sim 18000$ 2MASS sources, we selected those with high photometrical quality in the $J, H$, and $K$ s bands. Following Comerón et al. (2005), we looked for candidate YSOs using the parameter $q=(J-H)-1.83 \times\left(H-K_{\mathrm{s}}\right)$. This formula allows one to distinguish between giant and main-sequence stars, and sources with IR excess. Sources with $q<-0.15$ are classified as objects with infrared excess, revealing the presence of dusty circumstellar envelopes, i.e. candidate YSOs. After applying this criterion, we found 39 sources with IR excess. Figure 8 (upper panel) displays the color-magnitude (CM) diagram of these sources. The ZAMS from O3 to B5 type stars is displayed at the left of the diagram, assuming a distance of $2.0 \mathrm{kpc}$. Almost all sources with IR excess present visual absorption higher than 10 mag. Table A.1 summarizes the main parameters of these sources, i.e. galactic coordinates, designation, and $J, H$, and $K$ s magnitudes.

For IRAS and MSX sources, we searched for candidate YSOs following the criteria of Junkes et al. (1992) and Lumsden et al. (2002), respectively. In the case of the IRAS catalog, we found no sources that satisfied the mentioned criteria in the selected region, while in the case of the
MSX catalog, we found only one source at RA, Dec $($ J2000) = $\left(18^{\mathrm{h}} 18^{\mathrm{m}} 56^{\mathrm{s}} .4,-11^{\circ} 34^{\prime} 0{ }^{\prime} 5\right)$, candidate to compact H II region.

To complete the search for candidate YSOs around the optical nebula, we used the GLIMPSE 3D (2007-2009) cata$\log$ to perform a photometric study in the region. Considering only sources detected in the four Spitzer-IRAC bands, we found 8477 sources. To investigate the evolutionary stage of these sources, we have analyzed their location in a color-color diagram (Fig. 8, lower panel), following the color criteria from Allen et al. (2004). As expected, most sources seem to cluster around $(0,0)$. This region of the diagram contains mostly background/foreground stars and Class III sources without intrinsic IR excess. Sources in red occupy the Class II region $(0 \lesssim[3.6]-[4.5] \lessgtr 0.7,0.4 \lesssim[5.8]-[8.0] \lessgtr 1)$, and their IR excess might be produced by accretion disks around the stellar object. Sources in the domain of Class I objects $(0.7 \lessgtr[3.6]-[4.5]$, $1 \lesssim[5.8]-[8.0])$ are displayed in blue. In this case, the IR excess originates in circumstellar envelopes around the YSO.

In Fig. 7 we display the position of the candidate YSOs mentioned above. Many of them are projected outside Cloud 3, which suggests that these sources are background/foreground objects unrelated to the region of study. Among the candidate YSOs projected onto Cloud 3, 22 are 2MASS sources, 

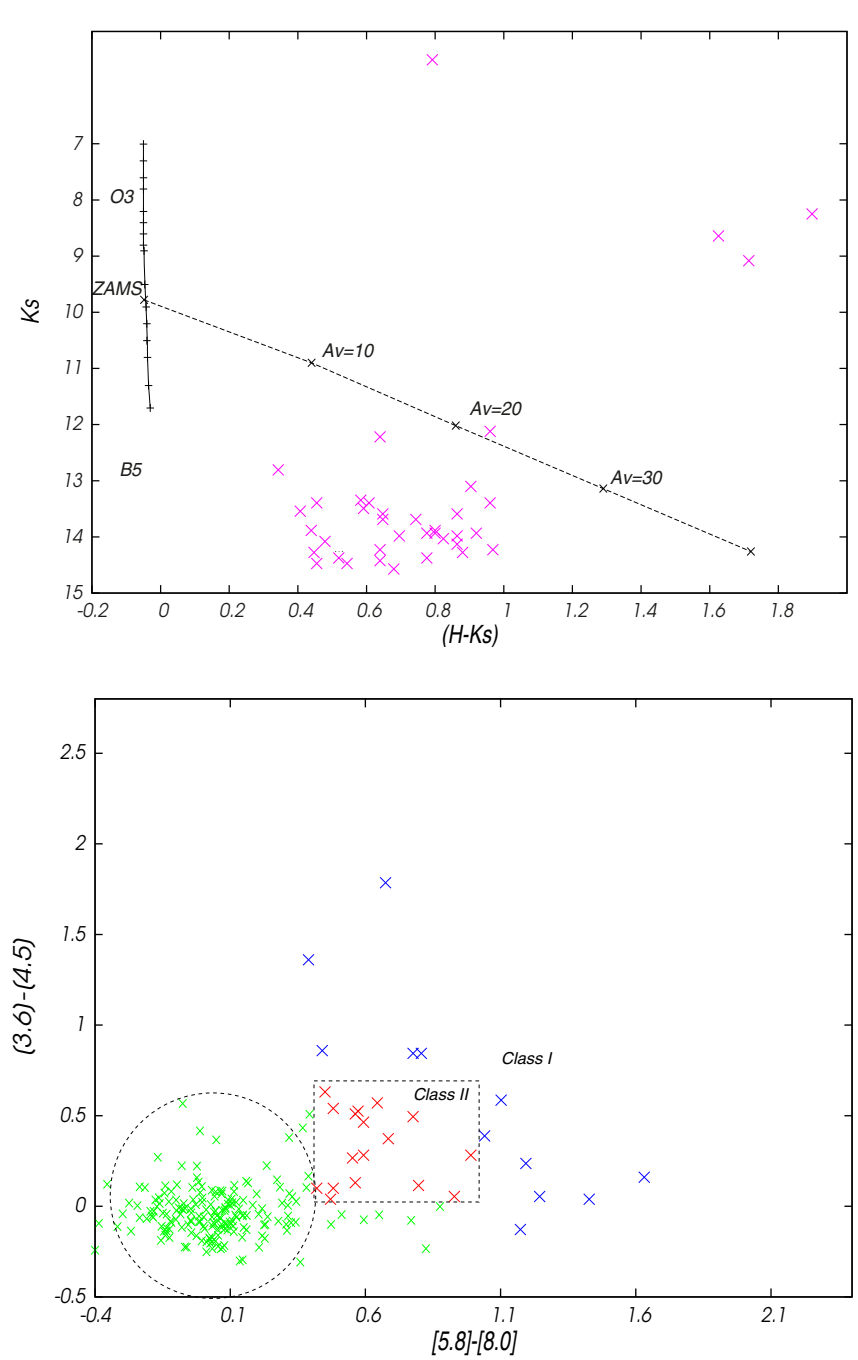

Fig. 8. Upper panel: color-magnitude diagram for the 2MASS sources with IR excess. Lower panel: color-color diagram for the Spitzer sources around the region of study.

while only six and four are Class I and Class II, respectively. Unlike the 2MASS sources, which are spread onto Cloud 3, Class I and Class II sources appear to be concentrated in two spots near RA, Dec $(J 2000)=\left(18^{\mathrm{h}} 18^{\mathrm{m}} 52^{\mathrm{s}},-11^{\circ} 38^{\prime} 45^{\prime \prime}\right)$. This location is almost coincident with a region of high optical absorption and a clump of molecular gas better noticed at velocities between +33.4 and $+35.2 \mathrm{~km} \mathrm{~s}^{-1}$ (see Fig. 4). This coincidence clearly shows that star formation is active in this region. The low value of the molecular mass $\left(\sim 1600 M_{\odot}\right)$ suggests that this cloud is the remnant of a larger parental cloud that has been photoionized and dissociated by the emerging young stars. To investigate whether these candidate Class I and Class II sources may have been triggered by the expansion of the molecular gas in a "collect and collapse" scenario, we applied the analytical model of Whitworth et al. (1994). According to this model, expanding nebulae compress gas between the ionization and the shock fronts, which leads to the formation of molecular cores where new stars can be embedded. Using Whitworth et al.'s formulae for expanding H II regions, we derived the time when the fragmentation may have occurred, $t_{\text {frag }}$, and the size of the HII region at $t_{\text {frag }}, R_{\text {frag }}$, which are given by $t_{\text {frag }}\left[10^{6} \mathrm{yr}\right]=1.56 a_{2}^{4 / 11} n_{3}^{-6 / 11} N_{49}^{-1 / 11}$ and $R_{\text {frag }}[\mathrm{pc}]=$ $5.8 a_{2}^{4 / 11} n_{3}^{-6 / 11} N_{49}^{1 / 11}$, where $a_{2}$ is the sound velocity in units of $0.2 \mathrm{~km} \mathrm{~s}^{-1}, n_{3} \equiv n_{\mathrm{H}_{2}} / 1000$, and $N_{49} \equiv N_{\text {Lyc }}^{*} / 10^{49}$. Adopting $0.3 \mathrm{~km} \mathrm{~s}^{-1}$ for the sound velocity (corresponding to temperatures of $3050 \mathrm{~K}$ in the surrounding molecular clouds), we obtained $t_{\text {frag }} \sim 1.2 \times 10^{6} \mathrm{yr}$, and $R_{\text {frag }} \sim 15 \mathrm{pc}$. Considering that $t_{\text {frag }}$ and $R_{\text {frag }}$ are higher than the dynamical age of Cloud $3\left(t_{\text {dyn }} \sim\right.$ $1.0 \times 10^{5} \mathrm{yr}$; see Sect. 6$)$ and its radious $\left(R_{\text {cloud } 3} \sim 2.3 \mathrm{pc}\right)$, we conclude that the fragmentation at the edge of Cloud 3 is doubtful. Nevertheless, the clustered aspect of these sources make them excellent candidates for investigating star formation with high angular resolution observations.

\section{Scenario}

As mentioned in Sect. 1.1, Gonzalez \& Rosado (1984) and Esteban \& Rosado (1995) (using optical lines), and Cappa et al. (2002) (using radio continuum data) explained the inner optical bright filament and its radio counterparts as the result of action of the UV field and the strong stellar winds of the star WR 113. An alternative scenario can be proposed taking into account the kinematical properties of Cloud 3, and the location of the WR star and SerOB2-1, -2, -3, -63, and -64.

Along the velocity interval from +32.5 to $+36 \mathrm{~km} \mathrm{~s}^{-1}$, WR 113 appears to be projected onto a region of faint molecular emission, which suggests that the star is interacting with the molecular cloud. The relatively wide velocity range of Cloud 3 $\left(\sim 7 \mathrm{~km} \mathrm{~s}^{-1}\right)$ suggests that expanding motions are present in the molecular gas. The classical wind-blown expanding shell scenario predicts that the surrounding gas expands spherically around the star. Then, if the molecular emission is a twodimensional projection of an expanding spherical shell, it should appear in the data cube first as a blueshifted pole (approaching cap), then as a growing-decreasing circular ring, and finally as a redshifted pole (receding cap), with the powering star placed at the center or close to it. According to Fig. 4, the approaching section of this shell is present, although the receding part is missing. Note also that the brightest optical ring coincides with the outer border of Cloud 3, indicating that this cloud is also being photodissociated by an external source, possibly located toward the southwest. Bearing in mind the location of SerOB-1, -2, -3, -63 and -64 (see Table 2) and their linear distance to Cloud 3 (1-2 pc, taking into account a distance of $2.0 \mathrm{kpc}$ ), we speculate that these stars are responsible for the inner optical ring. The diffuse optical emission observed north of the bright optical ring, in the region between $18^{\mathrm{h}} 18^{\mathrm{m}} 50^{\mathrm{s}}<$ RA $<18^{\mathrm{h}} 19^{\mathrm{m}} 20^{\mathrm{s}}$ and $-11^{\circ} 36^{\prime}<$ Dec $<-11^{\circ} 40^{\prime}$ might arise from the photodissociation of the molecular gas at the surface of Cloud 3 that faces the Earth, probably at lower radial velocities (between +30 and $+32 \mathrm{~km} \mathrm{~s}^{-1}$ ), since we indeed detect optical emission. The diffuse optical emission at the south of the optical rim, between $-11^{\circ} 40^{\prime}<$ Dec $<-11^{\circ} 42^{\prime}$ probably originates in the dissociation of the small molecular clump at RA, Dec $(\mathrm{J} 2000)=\left(18^{\mathrm{h}} 19^{\mathrm{m}} 3^{\mathrm{s}},-11^{\circ} 41^{\prime} 30^{\prime \prime}\right)$, with velocities between +34.3 and $+36.0 \mathrm{~km} \mathrm{~s}^{-1}$.

In Fig. 9 we present a side view of a simple sketch of the scenario proposed to explain the characteristics of the molecular and ionized gas. Cloud 3 is a half-shell that expands around CV Ser as the result of the wind mechanical energy injected into the ambient medium by the binary system. The stars SerOB2-1, $-2,-3,-63$, and -64 , belonging to SerOB2, photodissociate and ionize the southern and central region of the outer face of Cloud 3, giving rise to the diffuse emission observed at optical wavelengths. According to this scenario, the intensity of the optical emission is expected to rise as the thickness of the emitting 


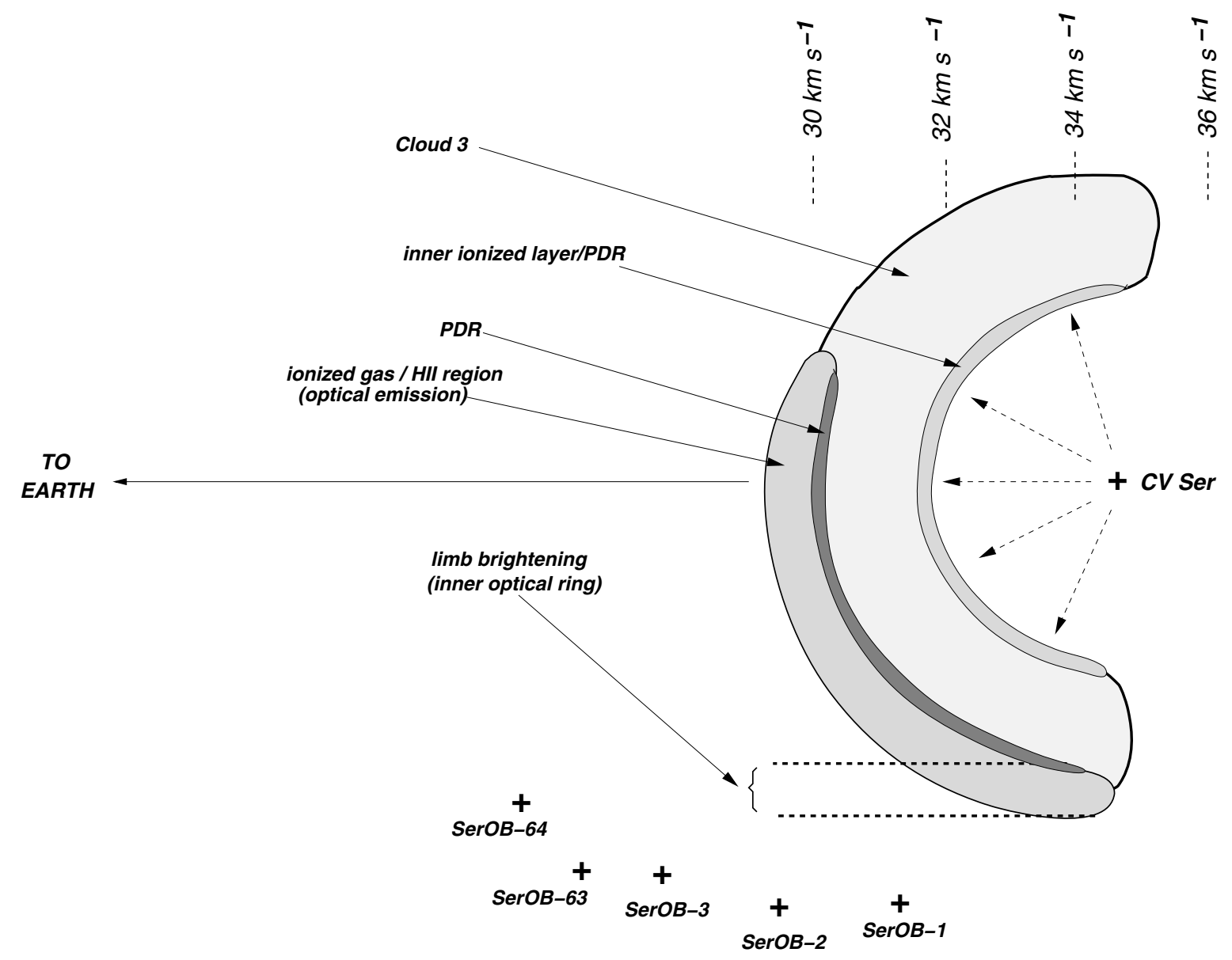

Fig. 9. Sketch of the model used to explain the characteristics of the molecular and ionized gas. The velocity field of Cloud 3 (see Fig. 4) is depicted in the upper right corner of the image.

ionized region in the line of sight increases. Therefore, the maximum of the optical emission should be observed toward the edge of the molecular cloud (limb brightening), which explain the location of the inner optical ring at the southern and western borders of Cloud 3 (see Fig. 9). The PAH emission originates in the PDR at the interface between the ionized and molecular gas. The UV field of CV Ser ionizes the inner face of Cloud 3, although the optical emission is probably mostly absorbed by the dust linked to the molecular gas in the line of sight.

We estimated an expansion velocity, $V_{\text {exp }}=6 \mathrm{~km} \mathrm{~s}^{-1}$. Similar expansion velocity values were derived for the expanding shells linked to WR stars, e.g. $\sim 7.4 \mathrm{~km} \mathrm{~s}^{-1}$ (Vasquez et al. 2009), $\sim 9 \mathrm{~km} \mathrm{~s}^{-1}$ (Cappa et al. 2009), $8 \pm 1 \mathrm{~km} \mathrm{~s}^{-1}$ (Vasquez et al. 2010). Adopting an expansion velocity of $6 \mathrm{~km} \mathrm{~s}^{-1}$, the dynamical age of the half-shell, according to wind-blown bubble models, can be calculated using $t_{\text {dyn }}=0.5 \times 10^{6} \times \frac{R}{V_{\text {exp }}}$ (McCray 1983; Howarth \& Lamers 1999), where $R$ is the radius of the bubble (pc). We obtain $t_{\text {dyn }} \approx 2 \times 10^{5} \mathrm{yr}$, which agrees, within the errors with the dynamical age obtained by Cappa et al. (2002) using radio continuum data.

From the value of the molecular mass and the expansion velocity, we determined a kinetic energy $E_{\text {kin }}=6 \times$ $10^{47} \mathrm{erg}$ and a momentum $P=9.6 \times 10^{3} M_{\odot} \mathrm{km} \mathrm{s}^{-1}$ for the molecular cloud. To calculate the mechanical energy injected by $\mathrm{CV}$ Ser, we assumed typical values for the stellar wind of a WC $8 \operatorname{star}\left(\dot{M}=2.5 \times 10^{-5} M_{\odot} \mathrm{yr}^{-1}, v_{\infty}=1890 \mathrm{~km} \mathrm{~s}^{-1}\right.$; Nugis \& Lamers 2000), and for its O9V type star companion
$\left(\dot{M}=4.0 \times 10^{-6} M_{\odot} \mathrm{yr}^{-1}\right.$ and $v_{\infty}=1800 \mathrm{~km} \mathrm{~s}^{-1}$; Smith et al. 2002). We obtain for CV Ser a mechanical luminosity of $L_{\mathrm{w}} \simeq$ $3.2 \times 10^{37} \mathrm{erg} \mathrm{s}^{-1}$. Considering a lifetime for the binary system equal to the age of Ser OB2 $\left(5 \times 10^{6} \mathrm{yr}\right.$; Forbes 2000$)$, the mechanical energy injected by the binary system into the interstellar medium is $E_{\mathrm{w}} \simeq 5.1 \times 10^{51} \mathrm{erg}$. Thus, the energy conversion efficiency is $\frac{E_{\mathrm{kin}}}{E_{\mathrm{w}}} \sim 0.01 \%$. In other words, only a small fraction of the mechanical energy released by CV Ser is needed to account for the kinetic energy of the expanding molecular gas. It is useful to stress that conversion efficiencies of $2-5 \%$ were reported in interstellar bubbles (Cappa et al. 2001; Cichowolski et al. 2001, 2003; Cappa et al. 2009). Furthermore, theoretical models and numerical simulations also suggest that in wind bubbles only a few percent of the injected mechanical energy will be converted into kinetic energy of the expanding gas (Weaver et al. 1977; Koo \& McKee 1992; Arthur 2007).

The derived values of energy conversion efficiencies are similar to estimates for other stellar wind bubbles (e.g., Cappa 2006). They agree with predictions from recent numerical simulations from Freyer et al. (2003) and (Freyer et al. 2006). The analysis by Freyer et al. takes into account the action of the stellar wind and the ionizing flux from stars of 35 and $60 M_{\odot}$ and find that $\frac{E_{\mathrm{kin}}}{E_{\mathrm{W}}}$ is in the range $0.10-0.04$. In addition, according to our model Cloud 3 subtends a small solid angle $(\lesssim 2 \pi)$, which reduces the wind mechanical energy available to it. Based on the above derived figures and considerations, the stellar winds of CV Ser are very capable of providing the kinetic energy of 
Cloud 3. Similarly, the momentum injected by the strong stellar wind of CV Ser to the ISM, taking into account $\dot{M}, v_{\infty}$ for the binary system is $P_{\mathrm{CVSer}} \approx 2.7 \times 10^{5} M_{\odot} \mathrm{km} \mathrm{s}^{-1}$, which yields a conversion efficiency $\frac{P}{P_{\mathrm{CVSer}}} \sim 4 \%$. The estimated value corresponds neither to the energy nor to the momentum conserving models. We believe that the existence of the aproaching molecular shell strongly affects the energy and momentum efficiencies.

\section{Summary}

With the aim of investigating the molecular gas around CV Ser and better understaning the interstellar scenario in the environs of the binary system, we analyzed SEST ${ }^{12} \mathrm{CO}(J=1-0)$ $\left(H P B W=44^{\prime \prime}\right)$ and $(J=2-1)\left(H P B W=22^{\prime \prime}\right)$ data, and complementary NANTEN ${ }^{12} \mathrm{CO}(1-0)$ data. The MIR data at 4.5 to $12 \mu \mathrm{m}$, along with Spitzer images allowed us to make a more comprehensive study of the dust around the nebula.

The more important aspects of this study can be summarized as follows:

- A molecular cloud in the velocity interval from $~+30$ to $+37 \mathrm{~km} \mathrm{~s}^{-1}$ (Cloud 3) was found to be associated with the optical ring nebula around the binary system CV Ser. Morphological and kinematical properties indicate that this cloud is a half shell expanding around CV Ser.

- The strong stellar winds of CV Ser are mainly responsible for shaping and for the kinematics of the molecular shell.

- The inner optical ring is placed along the outer edge of the molecular cloud. This suggests that the stars Ser OB2-1, -2, $-3,-63$ and -64 , belonging to SerOB2 and located 1-2 pc far from Cloud 3, ionize the outer face of the molecular cloud, generating the optical ring nebula and the diffuse optical emission observed toward the center of the cloud. The observed PDR located between the molecular cloud and the ionized gas is also generated by the action of these stars.

- A collection of candidate YSOs are detected toward the molecular cloud. Two small clusters of Class I and Class II objects are projected onto a region of high optical absorption, coincident with a small clump of molecular gas, indicating that star formation is active in this region. Although analytical models indicate that a triggered star formation scenario is doubtful, more studies are necessary to shed some light on this question.

Acknowledgements. We acknowledge the anonymous referee of her/his comments. J.V. acknowledges the hospitality of the Astronomy Department of Universidad de Chile during his stay in Santiago, Chile. We are grateful to Dr. N. Mizuno for providing us the NANTEN data. This project was partially financed by CONICET of Argentina under project PIP 2488/09, UNLP under project 11/G093, and ANPCyT under project PICT 903/08. M.R. is supported by the Chilean Center for Astrophysics FONDAP No. 15010003. M.R., J.V., C.C., and N.D. wish to acknowledge support from FONDECYT (Chile) grant No. 1080335. This research has made use of the NASA/IPAC Infrared Science Archive, which is operated by the Jet Propulsion Laboratory, California Institute of Technology, under contract with the National Aeronautics and Space Administration. This publication makes use of data products from the Two Micron All Sky Survey, which is a joint project of the University of Massachusetts and the Infrared Processing and Analysis Center/California Institute of Technology, funded by the National Aeronautics and Space Administration and the National Science Foundation.

\section{References}

Allen, L. E., Calvet, N., D’Alessio, P., et al. 2004, ApJS, 154, 363

Arthur, S. J. 2007, Wind-Blown Bubbles around Evolved Stars, eds. T. W. Hartquist, J. M. Pittard, \& S. A. E. G. Falle, 183

Benjamin, R. A., Churchwell, E., Babler, B. L., et al. 2003, PASP, 115, 953

Booth, R. S., Delgado, G., Hagstrom, M., et al. 1989, A\&A, 216, 315

Brand, J., \& Blitz, L. 1993, A\&A, 275, 67

Burton, W. B., \& Gordon, M. A. 1978, A\&A, 63, 7

Cappa, C. E. 2006, in Rev. Mex. Astron. Astrofis. Conf. Ser., 26, 9

Cappa, C. E., Rubio, M., \& Goss, W. M. 2001, AJ, 121, 2664

Cappa, C. E., Goss, W. M., \& Pineault, S. 2002, AJ, 123, 3348

Cappa, C., Niemela, V. S., Martín, M. C., \& McClure-Griffiths, N. M. 2005, A\&A, 436, 155

Cappa, C. E., Rubio, M., Martín, M. C., \& Romero, G. A. 2009, A\&A, 508, 759 Castets, A., Duvert, G., Dutrey, A., et al. 1990, A\&A, 234, 469

Chu, Y. H. 1991, in Wolf-Rayet Stars and Interrelations with Other Massive Stars in Galaxies, eds. K. A. van der Hucht, \& B. Hidayat, IAU Symp., 143, 349

Cichowolski, S., Pineault, S., Arnal, E. M., et al. 2001, AJ, 122, 1938

Cichowolski, S., Arnal, E. M., Cappa, C. E., Pineault, S., \& St-Louis, N. 2003, MNRAS, 343, 47

Comerón, F., Schneider, N., \& Russeil, D. 2005, A\&A, 433, 955

Conti, P. S., \& Vacca, W. D. 1990, AJ, 100, 431

Conti, P. S., Ebbets, D., Massey, P., \& Niemela, V. S. 1980, ApJ, 238, 184

Dickman, R. L. 1978, ApJS, 37, 407

Digel, S. W., Grenier, I. A., Heithausen, A., Hunter, S. D., \& Thaddeus, P. 1996, ApJ, 463, 609

Esteban, C., \& Rosado, M. 1995, A\&A, 304, 491

Forbes, D. 2000, AJ, 120, 2594

Freyer, T., Hensler, G., \& Yorke, H. W. 2003, ApJ, 594, 888

Freyer, T., Hensler, G., \& Yorke, H. W. 2006, ApJ, 638, 262

Gierens, K. M., Stutzki, J., \& Winnewisser, G. 1992, A\&A, 259, 271

Gonzalez, J., \& Rosado, M. 1984, A\&A, 134, L21

Goss, W. M., \& Day, G. A. 1970, Aust. J. Phys. Astrophys. Suppl., 13, 3

Howarth, I., \& Lamers, H. J. G. 1999, J. Brit. Astron. Assoc., 109, 347

Johansson, L. E. B., Greve, A., Booth, R. S., et al. 1998, A\&A, 331, 857

Junkes, N., Fuerst, E., \& Reich, W. 1992, A\&A, 261, 289

Koesterke, L., \& Hamann, W.-R. 1995, A\&A, 299, 503

Koo, B.-C., \& McKee, C. F. 1992, ApJ, 388, 93

Lamontagne, R., Moffat, A. F. J., Drissen, L., Robert, C., \& Matthews, J. M. 1996, AJ, 112, 2227

Leitherer, C., Forbes, D., Gilmore, A. C., et al. 1987, A\&A, 185, 121

Leitherer, C., Chapman, J. M., \& Koribalski, B. 1997, ApJ, 481, 898

Lockman, F. J. 1989, ApJS, 71, 469

Lumsden, S. L., Hoare, M. G., Oudmaijer, R. D., \& Richards, D. 2002, MNRAS, 336,621

Massey, P., \& Niemela, V. S. 1981, ApJ, 245, 195

McCray, R. 1983, Highlights Astron., 6, 565

Mueller, P., Reif, K., \& Reich, W. 1987, A\&A, 183, 327

Nugis, T., \& Lamers, H. J. G. L. M. 2000, A\&A, 360, 227

Nugis, T., Crowther, P. A., \& Willis, A. J. 1998, A\&A, 333, 956

Sakamoto, S., Hayashi, M., Hasegawa, T., Handa, T., \& Oka, T. 1994, ApJ, 425, 641

Sakamoto, S., Hasegawa, T., Handa, T., Hayashi, M., \& Oka, T. 1997, ApJ, 486, 276

Smith, L. J., Norris, R. P. F., \& Crowther, P. A. 2002, MNRAS, 337, 1309

Strong, A. W., \& Mattox, J. R. 1996, A\&A, 308, L21

Urquhart, J. S., Morgan, L. K., \& Thompson, M. A. 2009, A\&A, 497, 789

van der Hucht, K. A. 2001, New A Rev., 45, 135

van der Tak, F. F. S., \& van Dishoeck, E. F. 2000, A\&A, 358, L79

Vasquez, J., Cappa, C. E., \& Pineault, S. 2009, MNRAS, 395, 2045

Vasquez, J., Cappa, C. E., Pineault, S., \& Duronea, N. U. 2010, MNRAS, 405, 1976

Weaver, R., McCray, R., Castor, J., Shapiro, P., \& Moore, R. 1977, ApJ, 218, 377

Whitworth, A. P., Bhattal, A. S., Chapman, S. J., Disney, M. J., \& Turner, J. A. 1994, A\&A, 290, 421

Wright, E. L., Eisenhardt, P. R. M., Mainzer, A. K., et al. 2010, AJ, 140, 1868

Yamaguchi, N., Mizuno, N., Saito, H., et al. 1999, PASJ, 51, 775

Pages 12 to 13 are available in the electronic edition of the journal at http://www . aanda.org 


\section{Appendix A: 2MASS and Spitzer-IRAC point sources}

Table A.1 summarizes the $J, H$, and $K_{\mathrm{s}}$ magnitudes and the [3.6], [4.5], [5.8], and [8.0] IR fluxes for the 2MASS and Spitzer-IRAC YSO candidates, respectively, projected over the region.

Table A.1. YSO candidates projected over the region.

\begin{tabular}{|c|c|c|c|c|c|c|}
\hline \multicolumn{7}{|c|}{ 2MASS } \\
\hline Name & \multicolumn{2}{|c|}{ RA, Dec(J2000) } & $J[\mathrm{mag}]$ & $H[\mathrm{mag}]$ & \multicolumn{2}{|l|}{$K_{\mathrm{s}}[\mathrm{mag}]$} \\
\hline $18190736-1137591$ & $18: 19: 7$ & $-11: 37: 59.1312$ & 7.016 & 6.283 & \multicolumn{2}{|l|}{5.492} \\
\hline $18190884-1139265$ & 18:19:8 & $-11: 39: 26.5932$ & 15.859 & 14.684 & \multicolumn{2}{|l|}{13.888} \\
\hline $18185686-1134000$ & 18:18:56 & $-11: 34: 0.0192$ & 13.448 & 10.152 & \multicolumn{2}{|l|}{8.255} \\
\hline $18185863-1135413$ & $18: 18: 58$ & $-11: 35: 41.3448$ & 14.492 & 13.956 & \multicolumn{2}{|l|}{13.547} \\
\hline $18190584-1131161$ & 18:19:5 & $-11: 31: 16.1292$ & 16.194 & 15.234 & \multicolumn{2}{|l|}{14.556} \\
\hline $18185132-1138198$ & 18:18:51 & $-11: 38: 19.8348$ & 14.913 & 13.997 & \multicolumn{2}{|l|}{13.395} \\
\hline $18184741-1139346$ & $18: 18: 47$ & $-11: 39: 34.668$ & 14.347 & 11.825 & \multicolumn{2}{|l|}{9.379} \\
\hline $18185437-1134484$ & $18: 18: 54$ & $-11: 34: 48.4104$ & 15.831 & 14.457 & \multicolumn{2}{|l|}{13.591} \\
\hline $18185653-1133388$ & $18: 18: 56$ & $-11: 33: 38.808$ & 16.310 & 14.856 & \multicolumn{2}{|l|}{13.937} \\
\hline $18191623-1131588$ & $18: 19: 16$ & $-11: 31: 58.8$ & 15.439 & 14.014 & \multicolumn{2}{|l|}{13.106} \\
\hline $18191547-1132478$ & $18: 19: 15$ & $-11: 32: 47.832$ & 15.336 & 14.708 & \multicolumn{2}{|l|}{14.256} \\
\hline 18191209-1131439 & $18: 19: 12$ & $-11: 31: 43.9248$ & 13.528 & 13.162 & \multicolumn{2}{|l|}{12.820} \\
\hline $18184689-1140203$ & $18: 18: 46$ & $-11: 40: 20.37$ & 16.275 & 15.167 & \multicolumn{2}{|l|}{14.198} \\
\hline $18191326-1132294$ & $18: 19: 13$ & $-11: 32: 29.4576$ & 16.161 & 14.827 & \multicolumn{2}{|l|}{13.961} \\
\hline $18191548-1134327$ & $18: 19: 15$ & $-11: 34: 32.7216$ & 15.955 & 14.709 & \multicolumn{2}{|l|}{13.938} \\
\hline $18184570-1139594$ & $18: 18: 45$ & $-11: 39: 59.4864$ & 13.532 & 10.775 & 9.065 & \\
\hline $18190241-1139407$ & 18:19:2 & $-11: 39: 40.7556$ & 13.711 & 12.860 & 12.218 & \\
\hline $18190655-1137351$ & 18:19:6 & $-11: 37: 35.148$ & 14.757 & 13.902 & 13.322 & \\
\hline $18192507-1134108$ & $18: 19: 25$ & $-11: 34: 10.8876$ & 12.806 & 10.280 & 8.657 & \\
\hline $18184479-1141092$ & $18: 18: 44$ & $-11: 41: 9.2148$ & 15.483 & 14.647 & 13.955 & \\
\hline $18185316-1138319$ & $18: 18: 53$ & $-11: 38: 31.9632$ & 15.598 & 14.851 & 14.331 & \\
\hline 18184510-1135094 & $18: 18: 45$ & $-11: 35: 9.4632$ & 14.612 & 13.099 & 12.139 & \\
\hline $18191292-1133406$ & $18: 19: 12$ & $-11: 33: 40.6296$ & 14.524 & 13.864 & 13.407 & \\
\hline 18185691-1138354 & $18: 18: 56$ & $-11: 38: 35.4084$ & 14.700 & 14.329 & 13.891 & \\
\hline $18184716-1138575$ & $18: 18: 47$ & $-11: 38: 57.5988$ & 15.651 & 14.994 & 14.453 & \\
\hline $18193130-1136578$ & $18: 19: 31$ & $-11: 36: 57.8808$ & 15.991 & 15.031 & 14.388 & \\
\hline $18190185-1131418$ & 18:19:1 & $-11: 31: 41.8692$ & 16.039 & 15.142 & 14.363 & \\
\hline $18190169-1141155$ & $18: 19: 1$ & $-11: 41: 15.5688$ & 15.338 & 14.318 & 13.671 & \\
\hline $18184726-1133344$ & $18: 18: 47$ & $-11: 33: 34.4772$ & 15.087 & 14.519 & 14.044 & \\
\hline $18191809-1138460$ & $18: 19: 18$ & $-11: 38: 46.0824$ & 15.443 & 14.408 & 13.668 & \\
\hline $18185062-1132452$ & $18: 18: 50$ & $-11: 32: 45.294$ & 16.203 & 15.118 & 14.242 & \\
\hline 18184694-1138052 & 18:18:46 & $-11: 38: 5.2584$ & 15.518 & 14.927 & 14.469 & \\
\hline $18185224-1140231$ & $18: 18: 52$ & $-11: 40: 23.1708$ & 14.921 & 14.073 & 13.484 & \\
\hline $18190983-1140452$ & 18:19:9 & $-11: 40: 45.228$ & 16.219 & 14.999 & 14.138 & \\
\hline 18184996-1137399 & $18: 18: 49$ & $-11: 37: 39.9936$ & 15.938 & 14.338 & 13.379 & \\
\hline $18191577-1137174$ & $18: 19: 15$ & $-11: 37: 17.4504$ & 15.883 & 14.867 & 14.223 & \\
\hline $18185159-1136448$ & $18: 18: 51$ & $-11: 36: 44.8344$ & 15.644 & 14.755 & 13.955 & \\
\hline 18190499-1140419 & 18:19:4 & $-11: 40: 41.9808$ & 15.997 & 14.832 & 14.012 & \\
\hline 18192064-1140341 & $18: 19: 20$ & $-11: 40: 34.1004$ & 15.215 & 14.217 & 13.566 & \\
\hline & & IRAC & Class I) & & & \\
\hline Name & RA, & Dec(J2000) & [3.6] [mJy] & {$[4.5][\mathrm{mJy}]$} & {$[5.8][\mathrm{mJy}]$} & {$[8.0][\mathrm{mJy}]$} \\
\hline G018.8888+01.8312 & $18: 18: 47$ & $-11: 36: 41.6088$ & 1.389000 & 0.923700 & 2.600000 & 5.412000 \\
\hline G018.8152+01.8312 & $18: 18: 39$ & $-11: 40: 34.9572$ & 4.999000 & 6.915000 & 9.280000 & 10.660000 \\
\hline G018.8190+01.8261 & $18: 18: 40$ & $-11: 40: 31.3932$ & 2.304000 & 5.135000 & 10.280000 & 8.187000 \\
\hline G018.8158+01.8223 & 18:18:41 & $-11: 40: 48.1116$ & 8.123000 & 11.270000 & 15.860000 & 18.620001 \\
\hline G018.8550+01.7845 & $18: 18: 54$ & $-11: 39: 48.0924$ & 19.340000 & 15.520000 & 26.340000 & 43.950001 \\
\hline G018.8514+01.7792 & $18: 18: 54$ & $-11: 40: 8.3856$ & 0.895400 & 1.261000 & 2.747000 & 2.281000 \\
\hline G018.8540+01.8193 & 18:18:46 & $-11: 38: 52.0728$ & 4.180000 & 3.822000 & 4.604000 & 6.690000 \\
\hline G018.8626+01.8707 & $18: 18: 36$ & $-11: 36: 57.4668$ & 2.881000 & 9.610000 & 20.260000 & 21.110001 \\
\hline G018.8565+01.7810 & $18: 18: 55$ & $-11: 39: 49.2012$ & 2.993000 & 2.224000 & 6.030000 & 15.150000 \\
\hline G018.8569+01.7784 & 18:18:55 & $-11: 39: 52.3584$ & 3.836000 & 2.565000 & 4.646000 & 8.199000 \\
\hline G018.7823+01.8288 & $18: 18: 36$ & $-11: 42: 23.4252$ & 5.035000 & 5.525000 & 6.159000 & 9.447000 \\
\hline G018.9059+01.6493 & $18: 19: 29$ & $-11: 40: 56.3916$ & 1.625000 & 0.925700 & 1.329000 & 2.185000 \\
\hline
\end{tabular}


J. Vasquez et al.: The interstellar medium in the environs of WR 113

Table A.1. continued.

\begin{tabular}{lcccccc}
\hline \hline \multicolumn{7}{c}{ IRAC (Class II) } \\
\hline G018.7887+01.7997 & $18: 18: 43$ & $-11: 42: 52.6608$ & 11.310000 & 10.780000 & 10.240000 & 8.016000 \\
G018.7500+01.7978 & $18: 18: 39$ & $-11: 44: 58.5132$ & 1.633000 & 1.678000 & 1.464000 & 1.380000 \\
G018.8687+01.7831 & $18: 18: 55$ & $-11: 39: 6.9192$ & 6.715000 & 7.711000 & 9.600000 & 8.156000 \\
G018.7872+01.8240 & $18: 18: 37$ & $-11: 42: 16.1028$ & 3.466000 & 3.565000 & 3.959000 & 3.744000 \\
G018.7660+01.8084 & $18: 18: 38$ & $-11: 43: 49.5444$ & 12.800000 & 12.590000 & 11.850000 & 11.430000 \\
G018.7741+01.8274 & $18: 18: 35$ & $-11: 42: 51.7788$ & 3.781000 & 3.821000 & 3.752000 & 4.299000 \\
G018.7748+01.8111 & $18: 18: 39$ & $-11: 43: 17.2596$ & 1.671000 & 1.770000 & 1.929000 & 1.675000 \\
G018.7760+01.7794 & $18: 18: 46$ & $-11: 44: 7.3068$ & 2.620000 & 2.677000 & 2.298000 & 1.841000 \\
G018.7769+01.8381 & $18: 18: 33$ & $-11: 42: 24.696$ & 2.354000 & 2.556000 & 2.122000 & 2.144000 \\
G018.9792+01.7830 & $18: 19: 80$ & $-11: 33: 16.7724$ & 10.160000 & 9.544000 & 5.075000 & 2.799000 \\
G018.7823+01.8288 & $18: 18: 36$ & $-11: 42: 23.4252$ & 5.035000 & 5.525000 & 6.159000 & 9.447000 \\
G018.7723+01.7188 & $18: 18: 58$ & $-11: 46: 2.0496$ & 2.182000 & 2.357000 & 1.775000 & 0.922700 \\
\hline
\end{tabular}

